\title{
Molecular Limits to the Quantum Confinement Model in Diamond Clusters
}

\author{
T. M. Willey, ${ }^{1}$ C. Bostedt, ${ }^{2,3, *}$ T. van Buuren, ${ }^{1}$ J. E. Dahl, ${ }^{4}$ S. G. Liu, ${ }^{4}$ R. M. K. Carlson, ${ }^{4}$ L. J. Terminello, ${ }^{1}$ and T. Möller ${ }^{2,3}$ \\ ${ }^{1}$ Lawrence Livermore National Laboratory, Livermore, California 94550, USA \\ ${ }^{2}$ Hamburger Synchrotronstrahlungslabor Hasylab at DESY, Hamburg, Germany \\ ${ }^{3}$ Technische Universität Berlin, PN 3-1, Hardenbergstrasse 36, 10623 Berlin, Germany \\ ${ }^{4}$ MolecularDiamond Technologies, Chevron, P.O. Box 1627, Richmond, California 94802, USA
}

(Received 13 May 2005; published 7 September 2005)

\begin{abstract}
The electronic structure of monodispersed, hydrogen-passivated diamond clusters (diamondoids) in the gas phase has been studied with x-ray absorption spectroscopy. The data show that the bulk-related unoccupied states do not exhibit any quantum confinement. Additionally, density of states below the bulk absorption edge appears, consisting of features correlated to $\mathrm{CH}$ and $\mathrm{CH}_{2}$ hydrogen surface termination, resulting in an effective redshift of the lowest unoccupied states. The results contradict the commonly used and very successful quantum confinement model for semiconductors, which predicts increasing band edge blueshifts with decreasing particle size. Our findings indicate that in the ultimate size limit for nanocrystals a more molecular description is necessary.
\end{abstract}

DOI: 10.1103/PhysRevLett.95.113401

PACS numbers: $36.40 .-\mathrm{c}, 73.22 .-\mathrm{f}, 81.07 . \mathrm{Nb}$

Quantum confinement is a very successful model for understanding the electronic structure of nanometer size semiconductor structures such as quantum wells, nanocrystals, and clusters. Generally speaking it predicts that with decreasing particle sizes the band gap increases due to shifting of the band edges. So far, experiments were typically performed on deposited or embedded systems composed of at least several tens or hundreds of atoms. Now, with the isolation of higher diamondoids [1] a new class of clusters allows a study of quantum confinement in the molecular size limit. The first diamondoid, adamantane, consists of the smallest possible single cage unit excised from the diamond lattice, with the dangling bonds terminated by hydrogen atoms. Each subsequent diamondoid adds one additional face-fused cage. From a basic sciences point of view the isolated diamondoids are perfectly sizeselected and hydrogen terminated diamond (group IV semiconductor) clusters in the bulk crystal lattice configuration. This class of ideal structures has been the basis for virtually all electronic structure calculations on semiconductor nanocrystals. To date, however, these perfect nanometer-sized structures have never been available and thus have not been investigated. Experimental systems researched thus far exhibit either a size distribution or are charged for mass separation, possess ill-defined surface reconstruction or termination, and are typically deposited on substrates inducing interaction with their environment while under investigation.

Diamond plays a special role within the group IV semiconductors. Experimentally, deposited Si [2] and Ge [3] nanocrystals with sizes above $1 \mathrm{~nm}$ exhibit clear, theoretically confirmed quantum confinement effects. Meanwhile, reports show large and controversial quantum confinement effects for nanocrystalline diamond [4-6], yet no confinement is measured for $4 \mathrm{~nm}$ nanodiamond [7]. Theoretically, diamond nanocrystals have been predicted to exhibit significant quantum confinement effects for sizes below $1 \mathrm{~nm}$ with the highest occupied-lowest unoccupied molecular orbital (HOMO-LUMO) gap to increase up to $7.6 \mathrm{eV}$ [7-9], while the bulk diamond band gap is $5.47 \mathrm{eV}$ [9].

In this Letter we report x-ray absorption (XAS) measurements on diamondoids in the gas phase ranging from adamantane (one diamond cage) to cyclohexamantane (six diamond cages) as pictured in Fig. 1. Synchrotron-based XAS is ideal for determining electronic structure and bonding configuration in carbon materials [10]. Measurements in the gas phase allow experimental investigation of the ideal, interaction-free systems forming the basis for the theoretical models. To the best of our knowledge, the current experimental results in the molecular limit of ideal semiconductor nanocrystals yield unprecedented data against which competing theoretical models can be tested.

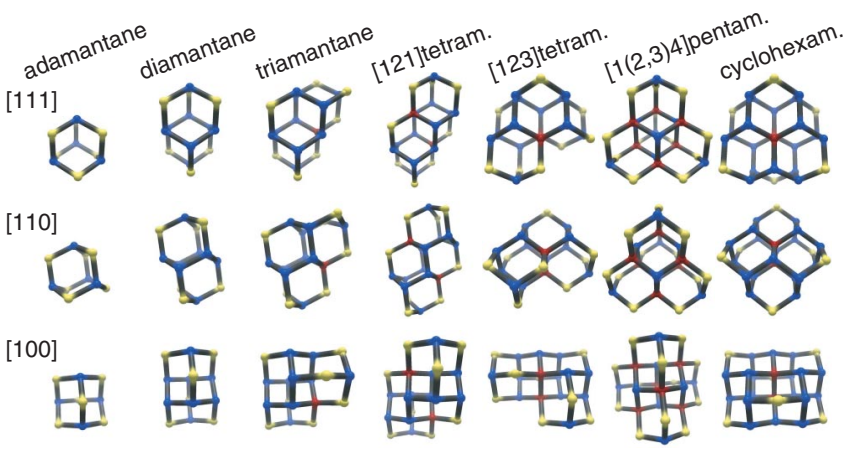

FIG. 1 (color online). Diamondoids investigated from adamantane to cyclohexamantane are shown in columns. The three rows presented view the diamondoids along various corresponding bulk lattice vectors. Colors indicate bulk-coordinated, $\mathrm{CH}$, and $\mathrm{CH}_{2}$ with red, blue, and yellow, respectively. 
The diamondoids are extracted from petroleum and size separated by high-performance liquid chromatography in ultrahigh purity [1]. Figure 1 shows structures of the investigated diamondoids adamantane, diamantane, triamantane, [121] tetramantane, [123] tetramantane, $[1(2,3) 4]$ pentamantane, and cyclohexamantane (using Balabanvon Schleyer structural nomenclature [11]). The diamondoids are perfectly size selected, are neutral, are fully $s p^{3}$ hybridized, and exhibit a complete hydrogen surface termination. This surface is of particular interest as such a hydrogen termination is used in most theoretical models describing semiconductor nanoclusters. Table I summarizes the stoichiometric formulas and atomic environments of the various diamond clusters. Only three basic configurations exist: carbon bound to only other carbon atoms (C), carbon bound to three other carbon atoms and one hydrogen $(\mathrm{CH})$, and carbon bound to two carbon and two hydrogen atoms $\left(\mathrm{CH}_{2}\right)$. In a bulk diamond picture, atoms $(\mathrm{C})$ can be interpreted as bulk-coordinated, whereas the atoms bound to hydrogen $\left(\mathrm{CH}\right.$ and $\left.\mathrm{CH}_{2}\right)$ can be considered surface atoms.

Fundamental geometric properties of investigated clusters can be inferred from Fig. 1 and Table I. The most basic diamondoids adamantane and diamantane exhibit only surface carbon atoms. Triamantane exhibits the first fully bulk-coordinated atom. Both isomers of tetramantane are stoichiometrically similar but [121] tetramantane exhibits a straight geometry while the [123] isomers, with left and right helicity, have a bent geometry. Pentamantane, a compact, tetrahedral structure, has a significant higher fraction of bulk-coordinated atoms than cyclohexamantane, which exhibits a disklike structure.

The $\mathrm{x}$-ray absorption measurements were performed at the high-resolution undulator beam line BW3 at the Hamburger Synchrotronstrahlungslabor HASYLAB at the Deutsches Elektronensynchrotron DESY in Hamburg (Germany). In brief, a monochromatized photon beam is crossed with the cluster beam under the aperture of a timeof-flight (TOF) spectrometer. The diamondoids are brought into the gas phase by sublimation at room temperature or by gentle heating up to $200^{\circ} \mathrm{C}$ with a temperature-stabilized in-vacuum furnace. The cluster beam is guided to the interaction region with a heated

TABLE I. Carbon binding environments in each diamondoid.

\begin{tabular}{lcccc}
\hline \hline & \multirow{2}{*}{ Chem. } & \multirow{2}{*}{ Bulk } & \multicolumn{2}{c}{ Surface } \\
& formula & $\mathrm{C}$ & $\mathrm{CH}$ & $\mathrm{CH}_{2}$ \\
\hline Adamantane & $\mathrm{C}_{10} \mathrm{H}_{16}$ & 0 & 4 & 6 \\
Diamantane & $\mathrm{C}_{14} \mathrm{H}_{20}$ & 0 & 8 & 6 \\
Triamantane & $\mathrm{C}_{18} \mathrm{H}_{24}$ & 1 & 10 & 7 \\
{$[121]$ tetramantane } & $\mathrm{C}_{22} \mathrm{H}_{28}$ & 2 & 12 & 8 \\
{$[123]$ tetramantane } & $\mathrm{C}_{22} \mathrm{H}_{28}$ & 2 & 12 & 8 \\
{$[1(2,3) 4]$ pentamantane } & $\mathrm{C}_{26} \mathrm{H}_{32}$ & 6 & 8 & 12 \\
Cyclohexamantane & $\mathrm{C}_{26} \mathrm{H}_{30}$ & 2 & 18 & 6 \\
\hline \hline
\end{tabular}

nozzle to avoid recondensation, and no carrier gas is used. For measuring the XAS spectra the photon energies are scanned through the $\mathrm{C} 1 s$ absorption edge. From direct ionization or autoionization as well as core hole decay processes, the clusters are charged and all ionic fragments are detected with the TOF. The recorded total ion yield approximates the absorption cross section and from the spectra conclusions about the atomic environment and electronic structure of the excited atom can be drawn [10]. The final state of the XAS process includes a core hole localized at the excited atom. Its influence will shift the energy levels on an absolute scale by multiple $\mathrm{eV}$ compared to ground state energies. However, as the core hole effect is similar for all diamondoids it does not affect trends and relative comparisons in a series of diamond clusters. Highly oriented pyrolytic graphite $\mathrm{C} K$-edge absorption was measured with the transmitted beam to calibrate energy scales. The beam line resolution was determined to be better than $0.1 \mathrm{eV}$. All spectra were normalized to the photon flux, measured with a highly transmissive $\mathrm{Au}$ grid. Pressures while acquiring the data were on the order of $10^{-6}$ mbar and the base pressure of the detector chamber was $10^{-8}$ mbar with partial pressures related to carbon contamination being in the $10^{-11} \mathrm{mbar}$ regime. Characterization of the adamantane beam with

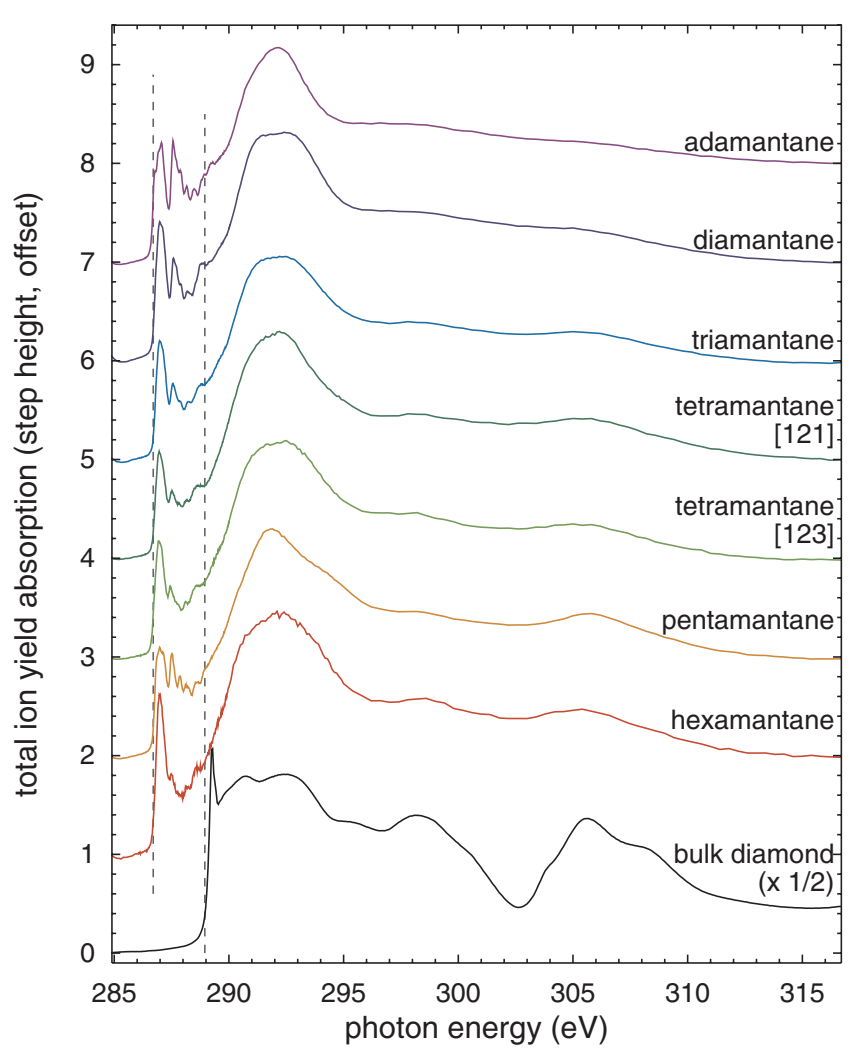

FIG. 2 (color online). The carbon $K$-edge absorption of the diamondoids adamantane to hexamantane, and a bulk diamond reference. 
mass spectrometery showed only single molecules and no larger aggregates.

Figure 2 presents the $\mathrm{C} K$-edge absorption data for the diamond cluster series from adamantane to cyclohexamantane and a diamond crystal reference. The spectra of the smallest diamondoids resemble previous XAS of the closely related cyclohexane [12] and earlier electron energy loss spectra (EELS) of gas-phase adamantane [13]. Through the series to larger sizes, though, the spectra already begin to exhibit the basic band structure of bulk diamond as the second absolute gap at $303 \mathrm{eV}$ emerges [14]. The diamondoids exhibit sharp spectral features starting at $286.7 \mathrm{eV}$, about $2.3 \mathrm{eV}$ below the absorption onset of the diamond reference sample. This spectral intensity is in the energy range of Rydberg, C-H valence, and mixed Rydberg-valence states of gaseous hydrocarbon molecules $[10,12]$. Furthermore, the features are comparable in energy to the intramolecular excitation within the $\mathrm{C}-\mathrm{H}$ bond of hydrogen terminated diamond (111) and (100) surfaces $[15,16]$. The left-hand panel of Fig. 3 presents the near edge structure from 286 to $290 \mathrm{eV}$ in more detail. The absorption onset is essentially the same for all clusters at $286.7 \mathrm{eV}$. Two manifolds of peaks, with an abundance of fine structure and separated by a gap at $287.4 \mathrm{eV}$, are labeled "area A" and "area B." Resolvable peak positions within these manifolds have energy spacings of 140-170 or $340-390 \mathrm{meV}$, and such modulation can be attributed to vibrational structure of the resonances [17]. The observed energy spacings are similar to $\mathrm{CH}$ and $\mathrm{CH}_{2}$ stretches and $\mathrm{CH}$ bending modes for condensed diamondoids determined by Raman and infrared absorption [18,19], and for hydrogenated diamond surfaces with high-resolution EELS [20]. Therefore the measured preedge features are

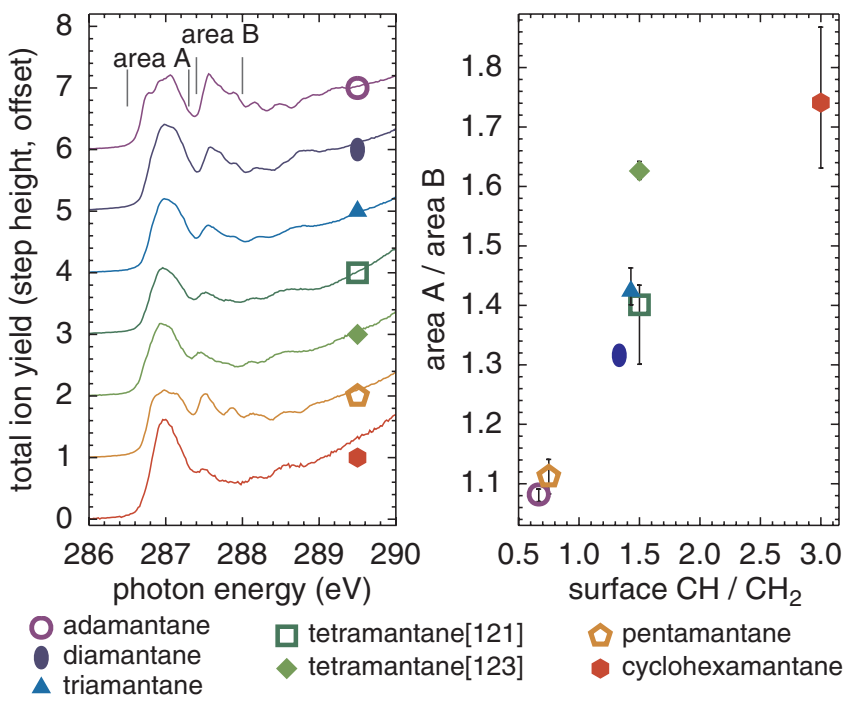

FIG. 3 (color online). Preedge carbon $K$-edge absorption spectra for the diamondoids adamantane to hexamantane (left) and empirical correlation between the manifolds labeled " $A$ " and " $B$ " with the two different surface species $\mathrm{CH}$ and $\mathrm{CH}_{2}$ (right). attributed to be primarily of $\mathrm{CH}$ valence character with clear vibrational structure and thus can be considered "surface states" of the diamond clusters. An empirical correlation between the features labeled "area A" and "area B" in the left-hand panel of Fig. 3 can be made with the number of $\mathrm{CH}$ to $\mathrm{CH}_{2}$ terminations (Table I) on each molecule. The right-hand panel of Fig. 3 presents the intensity of area A divided by area $\mathrm{B}$, vs the number of $\mathrm{CH}$ divided by $\mathrm{CH}_{2}$ terminations. A nearly direct linear dependence occurs between the two; therefore area $\mathrm{A}$ is primarily due to $\mathrm{CH}$ valence states, while area $\mathrm{B}$ is dominated by $\mathrm{CH}_{2}$ valence orbitals. It is interesting to note that [123] tetramantane deviates from this line and has an intensity which more closely resembles that of cyclohexamantane (see Fig. 1). This effect may be due to geometrical effects on these molecular orbitals, as these two diamondoids are very similar in shape. Additionally, steric hindrance of the additional $\mathrm{CH}_{2}$ terminations in the [123] tetramantane can also contribute to this effect.

Following the absorption edge in Fig. 2 towards higher photon energies, just beyond the bulk absorption onset, the preedge features merge into a broad resonance centered at $292 \mathrm{eV}$. These unoccupied densities of states (UDOS) can be attributed to the $\sigma^{\star}$ states of the C-C bonds in a molecular picture [10,12]. It is these interior $\sigma^{\star}$ states in the diamondoids (Fig. 2) that evolve through nanodiamond [7] to the bulk crystal (Fig. 2) where they constitute the first band including the conduction band minimum. For ease of reference, the $\mathrm{C}-\mathrm{C}$ bond related states therefore are referred to as bulk-related UDOS while the $\mathrm{CH}$ and $\mathrm{CH}_{2}$ related preedge features are referred to as surface related UDOS. In previous studies on size dependent electronic structure effects in semiconductor nanocrystals (e.g., experimental $[2,3]$, theoretical $[8,9])$ the bulk-related states exhibit the quantum confinement effects. While the $\sigma^{\star}$ resonance slightly changes shape in the transition from adamantane to cyclohexamantane with clear outliers of the compact adamantane and pentamantane structures, no clear blueshift of the lowest bulk-related empty states can be identified using the standard routines of extrapolation of the edge features to the baseline $[2,3]$ or fitting the absorption onset. This finding is independent of the cluster size or the number of bulk-coordinated atoms (compare Table I). The experimental results, namely, the nonshifted absorption edge and surface related preedge features dominating the LUMO, contradict the current understanding of quantum confinement, predicting blueshifted band edges with decreasing size down to the molecular limit.

The observed nonshifted absorption edge is further evidence for the special role of diamond within the group IV semiconductors. In earlier XAS investigations diamond nanocrystals $4 \mathrm{~nm}$ in size have no conduction band minimum shift [7], and theoretical predictions show clear quantum confinement only below $1 \mathrm{~nm}$ [7-9]. In comparison, the conduction band minima of $\mathrm{Si}$ and Ge nanocrystals 
of $1.2 \mathrm{~nm}$ in size were measured with XAS to shift by 0.4 [2] and $1.2 \mathrm{eV}$ [3], respectively, due to quantum confinement effects. Various calculations for small diamond cluster show the band gap to increase up to $7.6 \mathrm{eV}$ [7-9] compared to $5.47 \mathrm{eV}$ of the bulk crystal. The calculated band gap increase coupled with the observed nonshifted bulk-related absorption edge indicate that any quantum confinement effects in diamond must solely take place in the valence band, which will be investigated in a future work. Detailed calculations on the ground state electronic structure of the smallest diamondoids [9] predict increasing HOMO-LUMO gaps with decreasing size, i.e., gaps of $6.2,6.8,7.2$, and $7.6 \mathrm{eV}$ for decamantane, tetramantane, diamantane, and adamantane. The calculations show LUMO shifts up to $4 \mathrm{eV}$ between adamantane and decamantane and $1 \mathrm{eV}$ between adamantane and diamantane or $2 \mathrm{eV}$ and more between adamantane and tetramantane, respectively [9]. Such a shift would be very obvious in the absorption spectra (Fig. 2) but cannot be observed. It should be noted that the calculations do not unambiguously identify if surface or bulk species constitute the LUMO. In any case the predicted shifts are in contrast to our experimental results, namely, the nonshifted bulk-related absorption edge and additional surface related preedge features. A recent tight binding calculation [8] for larger clusters predicts no LUMO shift down to 34 carbon atoms, which is in good agreement with the bulk-related absorption edge of our largest investigated clusters (26 atoms). However, this and all other theoretical approaches [7-9] fail to predict the observed additional spectral features below the bulk absorption onset due to the hydrogen surface. These specific states, effectively reducing the overall particle gap, are fixed in energy and agree energetically with the hydrogen bonds of cyclohexane [12] in the molecular and diamond surface states $[15,16]$ in the solid state limit. The present experiments show that the hydrogen surface atoms have a significant impact on the overall electronic structure of the diamond clusters to a degree where they dominate the absorption onset and thus lowest unoccupied states.

In summary, we have measured the $\mathrm{x}$-ray absorption of perfect, free, and neutral diamond clusters ranging in size from adamantane to cyclohexamantane to gain insight into size dependent changes in the unoccupied states and the evolution of these states into the conduction band structure of diamond. Contradictory to other group IV semiconductors, the bulk-related absorption onset does not exhibit a size dependent blueshift as expected within the quantum confinement model. Furthermore the diamond clusters exhibit additional spectral intensity below the bulk absorption onset and within the band gap of bulk diamond, which can be empirically correlated to the $\mathrm{CH}$ and $\mathrm{CH}_{2}$ species of the surface termination. These states, dominating the absorption onset, have not been appropriately predicted in any of the current theoretical models. The experimental results show that these diamond clusters differ significantly from their related bulk diamond crystal and therefore require a more molecular description in their ultimate size limit.

We thank A. P. Hitchcock for valuable discussions and all Hasylab staff, particularly P. Gürtler, R. Döhrmann, and J. Brehling, for their outstanding support. This work was partially performed under the auspices of the U.S. DOE by the University of California Lawrence Livermore National Laboratory under Contract No. W-7405-ENG-48. C. B. acknowledges support from DESY/Hasylab.

*Corresponding author.

Electronic address: bostedt@physik.tu-berlin.de

[1] J. E. Dahl, S. G. Liu, and R. M. K. Carlson, Science 299, 96 (2003).

[2] T. van Buuren, L. N. Dinh, L. L. Chase, W. J. Siekhaus, and L. J. Terminello, Phys. Rev. Lett. 80, 3803 (1998).

[3] C. Bostedt, T. van Buuren, T. M. Willey, N. Franco, L. J. Terminello, C. Heske, and T. Möller, Appl. Phys. Lett. 84, 4056 (2004).

[4] Y. K. Chang et al., Phys. Rev. Lett. 82, 5377 (1999).

[5] L. Ley, J. Ristein, and R. Graupner, Phys. Rev. Lett. 84, 5679 (2000).

[6] W. F. Pong, M.-H. Tsai, and Y. K. Chang, Phys. Rev. Lett. 84, 5680 (2000).

[7] J.-Y. Raty, G. Galli, C. Bostedt, T. W. van Buuren, and L. J. Terminello, Phys. Rev. Lett. 90, 037401 (2003).

[8] D. A. Areshkin, O. A. Shenderova, S. P. Adiga, and D. W. Brenner, Diam. Relat. Mater. 13, 1826 (2004).

[9] G. C. McIntosh, M. Yoon, S. Berber, and D. Tománek, Phys. Rev. B 70, 045401 (2004).

[10] J. Stöhr, NEXAFS Spectroscopy, Springer Series in Surface Sciences (Springer-Verlag, Berlin, 1992).

[11] A. T. Balaban and P.R. von Schleyer, Tetrahedron 34, 3599 (1978).

[12] A. P. Hitchcock, D. C. Newbury, I. Ishii, J. A. Horsley, R. D. Redwing, A.L. Johnson, and F. Sette, J. Chem. Phys. 85, 4849 (1986).

[13] A.P. Hitchcock and I. Ishii, available in the Gas Phase Core Exitation Database, http://unicorn.mcmaster.ca/ corex/cedb-title.html (unpublished).

[14] J. F. Morar, F. J. Himpsel, G. Hollinger, G. Hughes, and J. L. Jordan, Phys. Rev. Lett. 54, 1960 (1985).

[15] R. Graupner, J. Ristein, L. Ley, and C. Jung, Phys. Rev. B 60, 17023 (1999).

[16] A. Hoffman, G. Comtet, L. Hellner, G. Dujardin and M. Petravic, Appl. Phys. Lett. 73, 1152 (1998).

[17] K. Ueda, J. Phys. B 36, R1 (2003).

[18] T. E. Jenkins and J. Lewis, Spectrochim. Acta 36A, 259 (1980).

[19] J.E. P. Dahl et al., Angew. Chem., Int. Ed. Engl. 42, 2040 (2003).

[20] A. Lafosse, D. Teillet Billy, J.-P. Guillotin, Y. Le Coat, R. Azria, A. Laikhtman, and A. Hoffmannn, Phys. Rev. B 68, 235421 (2003). 